\title{
Seismic Response Analysis of Crossing Pipeline in High-intensity Region
}

\author{
Zhen-chao Teng, Jia Zhao, Xiao-yan Liu and Tian-jia Zhao \\ School of Civil Engineering and Architecture, Northeast Petroleum University, \\ Daqing163318,China
}

\begin{abstract}
Keywords: Seismic Analysis; Modal Analysis; Crossing Pipeline; Analysis of Dynamic Characteristics
\end{abstract}

\begin{abstract}
In order to improve the seismic performance of structures, a crossing-pipeline model is established and the dynamic characteristics and seismic response are analyzed as well by adopting ANSYS finite element analysis and taking the practical pipeline project as an example. All of the research is based on the structure of long-distance pipelines. Then, the influence of thickness of pipeline wall, height and length of crossing pipelines on the pipelines is studied. The results show that on the premise that the pipe diameter is constant, the pipeline stability increases with the increasing of wall thickness and the decreasing of the height of pipes. The research can provide a reference for design of crossing-pipeline projects.
\end{abstract}

\section{Introduction}

There are many types of crossing pipelines which are suitable for crossing small rivers, as they are generally in simple type, it is convenient to construct and the cost is low as no framework is required. Moreover, it makes full use of the self-supporting ability of the pipeline. The research on crossing pipeline for the truss mainly highlights on the structural design of the truss and the weight of the pipeline is converted to the static load by adopting the mass of equivalence method and it subsequently exerted onto the truss, so the truss plays a supporting role. Taking the actual gas pipeline project as an example with the pipeline taken as the research object, the truss is converted into the constraint condition and exerted to the pipeline. In this regard, the dynamic characteristics and seismic responses of pipelines are studied[1-3].

\section{Establishment of Model and Selection of Seismic Wave}

\section{Establishment of Model}

The model of crossing pipelines is established by taking a practical pipeline engineering as an example and adopting the software of finite element analysis-ANSYS.The pipeline is made of Q235 steel with the density being $7.85 \mathrm{~kg} / \mathrm{m} 3$, elastic modulus being $210 \mathrm{GPa}$, Poisson ratio being 0.3 , external diameter of pipeline being $325 \mathrm{~mm}$. The specific parameters of model are shown in table 1 .

The pipeline is simulated by beam element BEAM189, divided by the free mesh and the bottom both ends of pipe are constrained by DOF in $\mathrm{x}, \mathrm{y}, \mathrm{z}$ the three directions and the partial node in the section of crossing pipeline is constrained by DOF in y direction. The pipeline model consists of 117 nodes and 114 elements. The finite element model is shown in figure 1 .

Table 1 Model parameter summary

\begin{tabular}{llllllll}
\hline model & A1 & A2 & A3 & B1 & B2 & C1 & C2 \\
\hline Wall thickness/mm & 15 & 16 & 18 & 15 & 15 & 15 & 15 \\
Height $/ \mathrm{m}$ & 6 & 6 & 6 & 4.5 & 3 & 6 & 6 \\
Span length/m & 21 & 21 & 21 & 21 & 21 & 27 & 33 \\
\hline
\end{tabular}




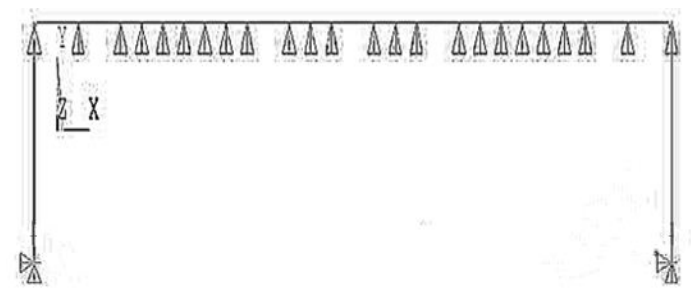

Fig.1Finite element calculation model

\section{Selection of seismic wave}

According to the Current China Seismic Design Code of Buildings GBJ11 [4], the EL-Centro seismic wave is selected, the interval is $0.02 \mathrm{~s}$, the duration is $20 \mathrm{~s}$, the acceleration in peak is 3.92 $\mathrm{m} / \mathrm{s} 2$. According to the standard, in the area with the basic seismic acceleration being $0.3 \mathrm{~g}$, the peak of the acceleration is $110 \mathrm{~cm} / \mathrm{s} 2$ when earthquake happens at 8 degree. Seismic wave must be multiplied by a certain adjustment factor and the versus acceleration curve of seismic wave after adjustment is shown in figure 2 .

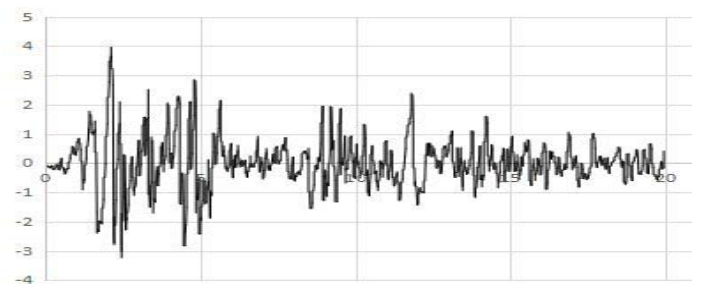

Fig.2 Selected seismic wave

\section{Analysis of Numerical Simulation Results}

\section{Modal analysis}

The response of dynamic characteristics for structure under seismic action is both related to seismic wave and the natural vibration characteristics of the structure. The natural frequency and formation of the structure are the base of calculation of structural seismic response and seismic design, therefore before the seismic analysis, the modal analysis is particularly necessary. The Block Lanczons method of ANSYS software is used to carry out analysis of formation for model $\mathrm{A} 1, \mathrm{~A} 2, \mathrm{~A} 3, \mathrm{~B} 1, \mathrm{~B} 2, \mathrm{C} 1$ and $\mathrm{C} 2$. The first ten natural frequencies of the model are obtained and the results are shown in table 2 .

Table 2Modal frequency summary

\begin{tabular}{cccccccc}
\hline model & $\mathrm{A} 1 / \mathrm{Hz}$ & $\mathrm{A} 2 / \mathrm{Hz}$ & $\mathrm{A} 3 / \mathrm{Hz}$ & $\mathrm{B} 1 / \mathrm{Hz}$ & $\mathrm{B} 2 / \mathrm{Hz}$ & $\mathrm{C} 1 / \mathrm{Hz}$ & $\mathrm{C} 2 / \mathrm{Hz}$ \\
\hline 1 & 0.000013 & 0.000017 & 0.009558 & 0.014 & 0.019 & 0.0103 & 0.000007 \\
2 & 0.967 & 0.970 & 0.971 & 1.457 & 1.942 & 0.908 & 0.805 \\
3 & 1.404 & 1.408 & 1.410 & 2.115 & 2.82 & 1.369 & 1.288 \\
4 & 3.565 & 3.576 & 3.581 & 5.3715 & 7.162 & 2.756 & 2.052 \\
5 & 8.914 & 8.940 & 8.954 & 13.431 & 17.908 & 7.410 & 5.538 \\
6 & 13.924 & 13.964 & 13.984 & 20.976 & 27.968 & 12.91 & 10.39 \\
7 & 16.368 & 16.411 & 16.433 & 24.6495 & 32.866 & 16.262 & 15.274 \\
8 & 17.061 & 17.111 & 17.136 & 25.704 & 34.272 & 16.46 & 15.732 \\
9 & 17.949 & 17.998 & 18.022 & 27.033 & 36.044 & 17.968 & 16.379 \\
10 & 20.085 & 20.141 & 20.169 & 30.2535 & 40.338 & 18.24 & 17.752 \\
\hline
\end{tabular}


From table 2, it is seen that: (1) first ten natural frequencies of the pipeline are between $0-41 \mathrm{~Hz}$ and they will increase with the increasing of frequencies, which is in linear change. (2) When the pipe diameter is constant, the natural frequency of pipeline increases with the increasing of thickness of walls. (3) Under the same conditions, with the decreasing of height, the natural frequency will increase and the higher the order is, the greater the degree will be, which is negatively proportional to the change of height. The height change is $6 \mathrm{~m}, 4.5 \mathrm{~m}, 3 \mathrm{~m}$, the ratio is approximately $4: 3: 2$. The change of natural frequency is approximately $2: 3: 4$. (4) In the same condition, the natural frequency of pipeline decreases with the increase of length.

\section{Seismic Analysis}

The Full method of transient analysis provided by ANSYS is used for seismic wave analysis. The corresponding maximum Von Mises stress and the maximum displacement are obtained. The results are shown in table 3 . The displacement and time curve in middle points of span $\mathrm{X}, \mathrm{Y}, \mathrm{Z}$ is shown in figure 3,4 and 5.

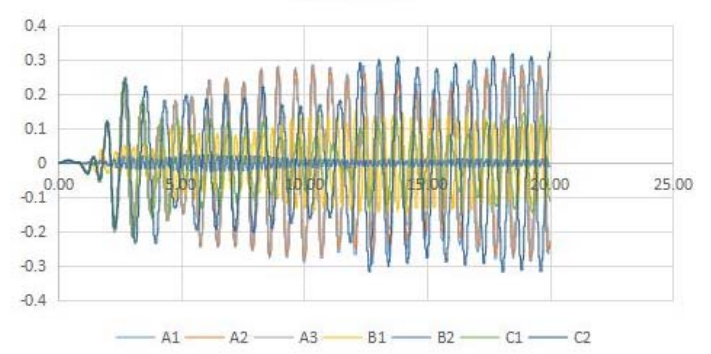

Fig. $3 \mathrm{X}$ direction shift of the mid span

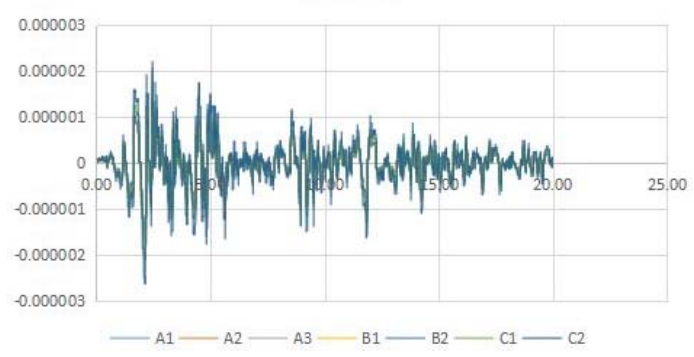

Fig.4 Y direction shift of the mid span

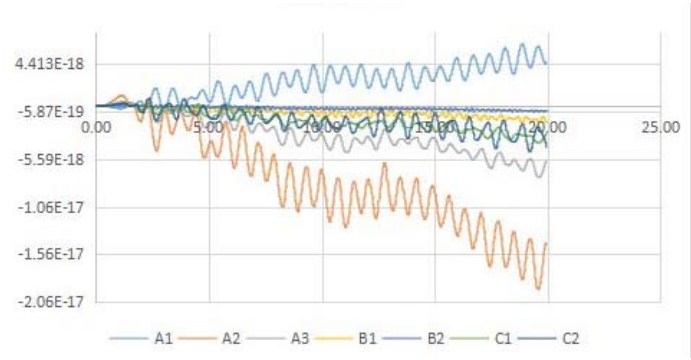

Fig.5 Z direction shift of the mid span

Table 3 Results summary

\begin{tabular}{lccccccc}
\hline \multicolumn{1}{c}{ model } & A1 & A2 & A3 & B1 & B2 & C1 & C2 \\
\hline maximum stress/Mpa & 278 & 249 & 188 & 228 & 183 & 294 & 411 \\
maximum displacement/cm & 29.36 & 27.05 & 22.35 & 15.41 & 2.79 & 29.98 & 32.48 \\
\hline
\end{tabular}

From table 3, it is known that: (1) When the pipeline diameter is a constant, the maximum stress 
and the maximum displacement decrease with the increasing of thickness of pipeline wall. (2) Under the same condition, the maximum stress and the maximum displacement decrease with the decreasing of pipeline height. (3) Under the same condition, the maximum stress and the maximum displacement increase with the increasing of length of span. (4) It is known that the yield limit of Q235 steel is 216-235 Mpa and according to the "Code for seismic design of oil (gas) steel pipeline" [5], the maximum stress of elastic plastic zone is $345 \mathrm{Mpa}$, the model A1,A2,C1is in plastic deformation, model A3,B1,B2is in elastic deformation and model $\mathrm{C} 2$ can be seen as being destructed.

\section{Conclusions}

The finite element model of pipeline is established by adopting ANSYS. After modal analysis and seismic response analysis are carried out, the following conclusions are obtained : (1) the higher natural frequencies can be obtained by increasing the thickness of walls, decreasing height and decreasing span and the probability of resonance is reduced. (2) Under the ceramic action, the height of wall of pipeline decreases and the deformation is transformed from elastic plastic deformation to elastic deformation, so the height of pipeline wall can effectively improve its seismic reserve. (3) In the engineering application, the length of span for single tube directly crossing the pipeline should not be too large.

\section{Acknowledgement}

This research was financially supported by the Key project of National Natural Science Foundation of China: Study on the basic problems of safe transportation of waxy crude oil pipeline. Item number:51534004

\section{References}

[1] Gao Peng, Wang Peihong, Wang Haiying and so on. Progress of Oil and Gas Pipeline Construction in China in 2014 [J] International Petroleum Economy2015,23(3):68-74,111,112

[2] Gao Jian, Wang Deguo, He Renyang and so on. Analysis of Seismic Versus Response for Cable-crossing Pipelines based on ANSYS [J] Journal of Southwest Petroleum Institute 2010,32(1):155-159

[3] Wang Haiyan, Zhao Ling, Wu Ming and so on. Finite Element Analysis of Crossing Pipeline Based on ANSYS [J]. Journal of Liaoning Shihua University. 2016,36(2):25-27

[4]GB50011-2010 Code for Seismic Design of Buildings[S] Beijing: People's Republic of China, Ministry of Construction, 2010

[5]SY/T0450-2004 Code for seismic design of oil (gas) steel pipelines[S] Beijing: People's Republic of China National Development and Reform Commission,2004 